\title{
Cold treatment enhances low-temperature flight performance in false codling moth, Thaumatotibia leucotreta (Lepidoptera: Tortricidae)
}

\author{
Nevill Boersma*†, Leigh Boardman ${ }^{\dagger *}$, Martin Gilbert ${ }^{\S}$ and John S. Terblanche ${ }^{\dagger}$ (D) \\ *XSIT (Pty) Ltd, 2 Schalk Patience Street, Citrusdal 7340, South Africa, ${ }^{\dagger}$ Centre for Invasion Biology, Department of Conservation Ecology and \\ Entomology, Stellenbosch University, Private Bag X1, Matieland 7602, South Africa, ${ }^{\ddagger}$ Department of Entomology and Nematology, University of \\ Florida, Gainesville, FL 32611, U.S.A. and ${ }^{\S}$ Citrus Research International, PO Box 28, Nelspruit 1200, South Africa
}

\begin{abstract}
In sterile insect technique programmes, temperatures experienced by insects during rearing and handling, along with cool temperatures after release, can negatively affect performance and activity levels. Phenotypic plasticity (trait modifications caused by prior stress exposure) can offset these effects but is poorly understood in many species and traits.

2 We investigated the effects of a cold treatment $\left(2{ }^{\circ} \mathrm{C}\right.$ for $\left.16 \mathrm{~h}\right)$ on flight performance in adult false codling moth, Thaumatotibia leucotreta. Using diverse methods, flight performance was tested using flight assays in the laboratory and in the field under varying environmental conditions.

3 The flight performance of T. leucotreta in the laboratory was affected by cold treatment (relative to a $25^{\circ} \mathrm{C}$ control group), test temperature and their interaction. Field recapture of released moths was significantly affected by the interaction between cold treatment and environmental conditions.

4 Field recapture counts depended on the ambient temperature upon release. For example, under warmer conditions $\left(>17^{\circ} \mathrm{C}\right)$, the recapture count of cold-treated moths was lower than that of the untreated control group, whereas the recapture count of cold-treated moths at cooler temperatures was significantly higher.

5 Our results suggest a temperature-dependent interaction between acute cold exposure and flight performance in adult $T$. leucotreta, which may be used to enhance the efficacy of the sterile insect technique under cooler environmental conditions.
\end{abstract}

Keywords acclimation response, activity thresholds, environmental effects, pest management, release-recapture, thermal history.

\section{Introduction}

The sterile insect technique (SIT) has been effectively used to suppress insect pests, some of which are vectors of human, plant and animal disease (Lindquist et al., 1992; Krafsur, 1998; Simmons et al., 2010). This technique uses mass rearing and non-lethal irradiation to sterilise insects in the laboratory. After sterilisation, insects are released to mate with wild conspecifics, resulting in non-viable offspring and leading to a decline in the target pest population (Dowell et al., 2005; Vreysen \& Robinson, 2010). Sterile insects have been used in integrated pest management programmes against various pests since the beginning of the 20th century. The most widely recognised

Correspondence: John S. Terblanche. Tel.:+27 (0)21 808 9225; fax: +27(0)21808 3235; e-mail: jst@sun.ac.za successful application of this technique is probably the eradication of the New World screwworm, Cochliomyia hominivorax Coquerel (Diptera: Calliphoridae), a parasitic fly that feeds on healthy flesh of animals (Klassen \& Curtis, 2005). Other examples include the pink bollworm, Pectinophora gossypiella (Saunders) (Lepidoptera: Gelechiidae), in the USA and codling moth, Cydia pomonella (Linnaeus) (Lepidoptera: Tortricidae), in Canada (Judd \& Gardiner, 2005).

A major challenge to the success of SIT is the competitiveness and field performance of mass-reared insects (Bloem et al., 1998, 2004; Judd \& Gardiner, 2006; Enserink, 2007; Terblanche \& Chown, 2007; Simmons et al., 2010; Sørensen et al., 2012). The SIT aims to achieve a high percentage of mating between the released sterile and natural populations to reduce reproduction to a level below an economic threshold. The production of 
high-quality and competitive individuals is therefore required to achieve this aim (Parker, 2005). In an SIT programme, thermal conditions during or after insect development may affect various traits, such as body size, fecundity, longevity, flight performance and mating competitiveness (Sørensen et al., 2012; Terblanche, 2014; Boersma \& Carpenter, 2016; Boersma et al., 2018). Typically, SIT programmes use optimal rearing temperatures to maximise production regardless of post-release environmental conditions, but this approach may negatively impact the dispersal potential of the mass-reared insects upon release (Bloem et al., 2004; Chidawanyika \& Terblanche, 2011; Sørensen et al., 2012; Mateev et al., 2017).

Treatment at cold temperatures is often used as a standard procedure to immobilise insects before release. Such cold treatment eases the collection, handling, irradiation, transportation and release of these insects. However, chilling and long-term cold-temperature storage may adversely impact moths' field performance if negative effects persist after rewarming and release, possibly leading to poorer performance or competitiveness than that of their wild counterparts (Boersma \& Carpenter, 2016). On the other hand, insects cold-treated prior to release may disperse and perform better under cooler field conditions through plastic physiological adjustments (i.e. beneficial acclimation). The adaptive value of physiological change from beneficial acclimation is widely debated in the evolutionary physiology literature although the effects are typically widely reported across diverse traits and species (see e.g. Wilson \& Franklin, 2002; Woods \& Harrison, 2002; Chown \& Terblanche, 2007). Nevertheless, beneficial acclimation remains valuable to SIT programmes seeking to manipulate insect field performance (Judd \& Gardiner, 2006; Chidawanyika \& Terblanche, 2011; Sørensen et al., 2012). Briefly, beneficial acclimation is thought to give an organism a performance advantage over another organism that was not acclimated to that particular environment (Leroi et al., 1994; Wilson \& Franklin, 2002). The physiological response to a particular environmental stressor may enhance the performance and fitness of insects during subsequent sub-optimal conditions, since they are able to continue performing vital activities, such as mating, feeding and flying that would otherwise not have been possible (Fasolo \& Krebs, 2004; Powell \& Bale, 2005).

Acclimation can occur over longer exposures to sub-optimal temperatures whereas hardening, defined as a rapid response to extreme or acute environments, can be achieved over shorter, typically more acute periods (Shreve et al., 2004; Denlinger \& Lee, 2010; Ju et al., 2013; Colinet et al., 2015). No consistent, systematic treatment conditions elicit a clear distinction between acclimation and cold hardening responses across species (see discussion in Teets \& Denlinger, 2013). For example, Bactrocera tryoni (Froggatt) (Diptera: Tephritidae) was found to acclimate in $2 \mathrm{~min}$, whereas Bactrocera oleae (Rossi) required several days (Meats, 1976; Fletcher \& Zervas, 1977; see discussion in Weldon et al., 2011). Treatment temperature and exposure time therefore have species-specific physiological effects that are themselves the focus of much attention (Shreve et al., 2004; Denlinger \& Lee, 2010; Ju et al., 2013; Colinet et al., 2015). Furthermore, since insects' tolerance to environmental conditions depends on the timing and duration of their exposure to prior environmental stress (Sgrò et al., 2016), thermal history in SIT programmes may affect competition between field and sterile mass-reared individuals, since multiple behaviour and locomotor performance traits can be strongly modified by thermal history (e.g. Kristensen et al., 2008; Chidawanyika \& Terblanche, 2011; Esterhuizen et al., 2014). Because of the complex range of responses to thermal stress, further investigation is needed to provide insight into responses of field-released insects and how they might be manipulated (Terblanche, 2014).

The false codling moth, Thaumatotibia leucotreta (Meyrick), is a phytosanitary pest of citrus fruit in southern Africa (Bloem et al., 2004). An SIT programme in citrus orchards was established in Citrusdal, Western Cape, South Africa, in 2007 (Hofmeyr et al., 2015). Sterile male and female T. leucotreta adults are released during the citrus growing and harvesting season, from spring to early winter (September to June) (Hofmeyr et al., 2005). Since the initiation of the programme, the wild T. leucotreta population has undergone a ten-fold decline. Pre-harvest crop losses due to T. leucotreta have also been reduced by $93 \%$ and post-harvest rejection by $38 \%$ (Hofmeyr et al., 2015). Despite the broad success of the commercial SIT programme, moth performance at low temperatures remains a challenge as sterile moths reared via a constant optimum regimen exhibit reduced flight activity under cool environmental conditions (Stotter \& Terblanche, 2009).

The thermal history of mass-reared T. leucotreta has a marked impact on a range of traits, such as longevity, fecundity and cold tolerance, with the extent of the impact varying between the sexes (Boersma et al., 2018). Sterile T. leucotreta adults have a minimum activity threshold temperature of approximately $10-15^{\circ} \mathrm{C}$, indicating reduced cold tolerance relative to the field conditions in which they must perform during colder months in spring and autumn $\left(5-15^{\circ} \mathrm{C}\right)$ (Stotter \& Terblanche, 2009). Similar effects have been observed in other SIT programmes involving the codling moth in Canada (Judd et al., 2004). Thermal preconditioning is viewed as a possible solution to poor post-release performance at low temperatures in various species (Fay \& Meats, 1987; Chidawanyika \& Terblanche, 2011; Sørensen et al., 2012; Terblanche, 2014).

We aimed here to determine the effect of thermal history on the flight performance of $T$. leucotreta under different thermal conditions. We focused on the effect of pre-release adult cold treatment (i.e. short-term exposure to cold temperatures) on the subsequent laboratory and field flight performance of the same cohort of moths. Specifically, we tested the hypothesis that short-term, acute exposure of sterile $T$. leucotreta adults to low temperatures prior to release will enhance flight performance, and hence recapture counts, under post-release cold conditions. This would indicate that plastic thermal physiology benefits flight performance. However, the benefits thereof are also likely dependent on the prevailing thermal conditions and we thus consider the environmental conditions during release in detail. The implications of these results are discussed in the context of enhancing the flight performance of T. leucotreta in an SIT programme.

\section{Materials and methods}

Insect rearing

The T. leucotreta culture used in this study was established in 2006. This laboratory population from the XSIT (Pty) Ltd mass-rearing facility (Citrusdal, Western Cape, South Africa) 
was established from wild moths collected in orange orchards $( \pm 6000 \mathrm{ha})$ from different regions and microclimates in the Elephants River Valley. The colony was augmented with wild insects at several intervals to prevent inbreeding depression or genetic divergence from natural populations. The population was maintained at $25 \pm 1{ }^{\circ} \mathrm{C}$ under a 12:12 (L:D) photoperiod on a maize-based diet. Relative humidity was not strictly controlled and ranged between 40 and 60\% (101A, MadgeTech, Warner, New Hampshire, USA). The temperature during the handling and transportation of adults prior commercial release was between 8 and $15^{\circ} \mathrm{C}$ under dark conditions.

\section{Treatments}

Moths for both laboratory and field flight performance assays were obtained from the above-mentioned commercial colony. Moths were collected from the moth emergence cabinets within $12 \mathrm{~h}$ post eclosion at $25^{\circ} \mathrm{C}$. Both male and female moths were used for laboratory assays, as previous research has shown significant differences between the sexes with regards to field traits, such as cold tolerance, fecundity and longevity (Boersma et al., 2018). For field flight performance however, only males were tested, as pheromone traps were used to monitor flight in the field.

Immediately after collection, all moths were irradiated with $150 \mathrm{~Gy}$ in a panoramic cobalt-60 source (double encapsulated C132 type 78 source; dose rate at $3.75 \mathrm{~Gy} / \mathrm{min}$ ) in a temperature-controlled room $\left(15 \pm 1^{\circ} \mathrm{C}\right)$, after which they were kept at $25^{\circ} \mathrm{C}$ for $20 \mathrm{~min}$ before they were either cold-treated overnight at $2 \pm 1{ }^{\circ} \mathrm{C}$ for $16 \mathrm{~h}$ in an environmental chamber (Binder climate chamber, KBW 240 Binder, Tuttlingen, Germany) or kept as a control group at $25 \pm 1{ }^{\circ} \mathrm{C}$ in a temperature-controlled room (confirmed using a temperature data logger, 101A, MadgeTech). A cold-treatment temperature of $2{ }^{\circ} \mathrm{C}$ was chosen to ensure that moths were kept below their critical thermal minimum (CTmin) $\left(\sim 6^{\circ} \mathrm{C}\right)$ (Terblanche et al. 2017) but above sub-zero temperatures to prevent ice formation. The chosen treatment temperature was deemed appropriate, as the current handling and transportation temperatures of the SIT operation have been associated with poor recapture counts under both warm and cold environmental conditions (Boersma \& Carpenter, 2016; Stotter \& Terblanche, 2009). Because commercially reared adults are kept in the dark after eclosion in the SIT facility, treatments were conducted overnight in complete darkness for $16 \mathrm{~h}$ (17:00-9:00) to simulate the facility's current handling and transportation protocols and procedures.

On the morning after treatment, both the treated and control groups were marked with $0.1 \mathrm{~g} / 100$ moths of differently coloured fluorescent micronized dust (DayGlo Color Corp., Cleveland, Ohio, USA) to allow distinction between treated and control moths, after which they were kept at $25 \pm 1{ }^{\circ} \mathrm{C}$ for $60 \mathrm{~min}$ to recover. Treatment colours were randomised between groups (i.e. colours were randomly assigned to either group) on each experimental day to eliminate any influence of dye colour on recapture counts. After recovery, the flight performance of moths was assessed in the laboratory or field, as described in the sections below. Scoring of recaptured moths was done using a UV light in a dark room at the laboratory to differentiate between the coloured markings.

\section{Laboratory flight performance}

For laboratory assays, a total of 720 moths (360 males and 360 females) were separated from the XSIT colony. Of these, 180 males and 180 females were subjected to the cold treatment as described in the treatment section above and equal numbers were kept as a control.

Laboratory flight performance assays were conducted under two different temperature conditions, namely $15 \pm 1{ }^{\circ} \mathrm{C}$ (cold) and $30 \pm 1{ }^{\circ} \mathrm{C}$ (warm), from September 2015 to June 2016. The sexes were tested separately, and the control and treated groups of each sex were tested together under each temperature condition. For each assay, $30 \mathrm{~T}$. leucotreta adults of the same sex from the control and cold-treated groups respectively, (i.e. 30 from the control and 30 from the cold-treated group), were placed in two separate Petri dishes (diameter $=45 \mathrm{~mm}$, height $=15 \mathrm{~mm}$ ). These Petri dishes were placed on a pedestal in a round water basin $(r=410 \mathrm{~mm})$ in a dark, temperature-controlled room $(3 \times 4 \times 1.8 \mathrm{~m})$ set to either 15 or $30^{\circ} \mathrm{C}$. The two test temperature conditions were simulated via an air-conditioning system in the room. The air temperature was monitored with a digital thermometer (Fluke II, Fluke Corporation, Everett, Washington, USA) connected to a type T thermocouple (36 standard wire gauge) and recorded at an interval of $1 \mathrm{~Hz}$ during all experiments. A blue fluorescent light (12 W Hand Scanner UV Light with UV Filter, Equip, Johannesburg, South Africa) was placed 2 m away from the basin to attract the moths to fly over the water obstacle. Moths were left to fly for $2 \mathrm{~h}$, after which each individual was categorised according to its dispersal performance, namely 'sustained flyer' (those that flew from the Petri dish to the light), 'partial flyer' (those that landed in the water) and 'non-flyer' (those that remained at the release site). The control and cold-treated moths were distinguished based on their dye markings. Each assay under each temperature condition for each sex was replicated three times. No moths were reused between assays.

\section{Field flight performance}

Field release-recapture experiments were performed in a citrus orchard $5 \mathrm{~km}$ from the rearing facility in Citrusdal. Thirty yellow delta traps containing sex pheromone lures ((E)-8-, (Z)-8- and (E)-7-dodecenyl acetate; CHEMPAC $®$ false codling moth, Paarl, South Africa) and sticky trap liners (CHEMPAC) for recapture were set up. The traps were placed in three groups of 10 rows of orange trees each with three rows of trees between each group. The traps were placed $6 \mathrm{~m}$ apart in the tenth tree from the border of each row, on the eastern side of the tree to distribute the sex pheromones into the orchard. The traps were suspended $1.5 \mathrm{~m}$ above the ground in the outer foliage of the trees. Leaves and twigs around the traps were removed to ensure unobstructed air flow and unhindered access for moths.

Upon eclosion, $600 \mathrm{~T}$. leucotreta male moths per replicate were separated from the XSIT colony and divided equally between two Petri dishes (diameter of $70 \mathrm{~mm}$ ). All moths were irradiated, after which 300 were cold-treated overnight, as described in the treatment section above, while the remaining 300 were kept as a control. Moths were marked as described in the treatment section above. These moths were transported to the test orchard in separate Petri dishes stored in an insulated box at $15^{\circ} \mathrm{C}$. Although T. leucotreta adults are nocturnal, moths were evenly 
Table 1 Additional climatic data and environmental conditions associated with orchard conditions for $72 \mathrm{~h}$ after release

\begin{tabular}{lll}
\hline & $\begin{array}{l}\text { Measurement } \\
\text { duration per day }\end{array}$ & Unit \\
\hline Average maximum night temperature & $18: 00-06: 00$ & ${ }^{\circ} \mathrm{C}$ \\
Absolute maximum night temperature & & \\
Average minimum night temperature & & \\
Absolute minimum night temperature & & \\
Mean night temperature & & \\
Cumulative number of hours below $6{ }^{\circ} \mathrm{C}$ & & $\%$ \\
Cumulative number of hours below $10{ }^{\circ} \mathrm{C}$ & & $\mathrm{Mm}$ \\
Average maximum relative humidity & 3 days & $\mathrm{m} / \mathrm{s}$ \\
Average minimum relative humidity & & $\mathrm{Mm}$ \\
Average total evaporation & & $\mathrm{MJ} / \mathrm{m}^{2}$ \\
Average wind speed & & \\
Total rainfall & & \\
Average total radiation & &
\end{tabular}

released by hand (approximately 10 cold-treated and 10 control moths per row) at 10:00 to simulate commercial SIT releases for all 30 rows, from a position $20 \mathrm{~m}$ north of the traps and at the end of each row.

The traps were left undisturbed for 3 days, after which the sticky trap liners were collected and the number of fluorescent T. leucotreta individuals per trap was counted. New sticky trap liners and pheromone lures were placed in the traps at the beginning of each trial. This was repeated over two seasons, namely seven times in late spring to early summer (18 September to 30 October 2015) and seven times in winter (3 June to 16 August 2016), to ensure that successful release-recapture trials were performed at both cooler and warmer temperatures. An electronic temperature data logger, set to log every hour (accuracy $\left.= \pm 0.1^{\circ} \mathrm{C}\right)(101 \mathrm{~A}$, MadgeTech), was placed centrally between the branches of a tree in the orchard to monitor air temperature. Additional data on the climate and environmental conditions associated with the orchard (Table 1) were obtained from the Citrusdal North weather station (Agricultural Research Council) approximately $5 \mathrm{~km}$ away from the test orchard for three nights and days after release. The total number of released moths for the trial was 9000 .

\section{Statistical analyses}

\section{Laboratory assays}

For the laboratory assays, we determined which of the four independent variables (sex, treatment, test temperature and biological replicate) played a role in the flight performance of $T$. leucotreta and whether any interactions between these variables existed. The effect of the independent variables on the dependent variable (dispersal) was tested against the dependent variable, comprising specific categories, namely non-, partial and sustained flyers, with non-flyers being used as a baseline category. Analyses were conducted using R software (v. 3.0.3; R Foundation for Statistical Computing, 2008, Vienna, Austria; packages 'nnet' and 'mlogit'). Data were analysed as the total number of recaptures for each of the three replicates under each temperature condition for each sex and treatment group. Differences in flight performance between sustained, partial and non-flyers and those between the treatment and control groups were determined using a multinomial logit model. This model was preferred over the ordinal multinomial regression, as the latter violated the proportional odds assumption. Furthermore, the nominal multinomial model explains the relative likelihood of being in one category versus being in the reference category (the log ratio of the variables) using a linear combination of predictor variables. Consequently, the probability of each outcome is expressed as a nonlinear function of the predictor variables. In this study, a distributional assumption was made in terms of the dependent variables. Subsequently, the mean performance was linked to a linear function of the predictors through a generalised logit link function (Field et al., 2012; Qian et al., 2012).

\section{Field assays}

The effect of the cold or control treatment on male moths was investigated and the importance and role of each independent variable (Table 1) in terms of the field flight performance of T. leucotreta was determined. Analyses were conducted using R software v. 3.0.3 (packages 'nnet' and 'randomForest') and figures were drawn in Statistica (v. 13.3; Statsoft Inc., Tulsa, Oklahoma, U.S.A.). Because environmental factors could be closely correlated, random forest analyses were conducted to determine which independent environmental factor contributed the most to the flight performance of T. leucotreta adults (Liaw \& Wiener, 2002; Chen \& Ishwaran, 2012). Using a stepwise ascending variable strategy, we determined the average decline in accuracy of each variable with an iterative algorithm that fits a different regression model at each node and over each iteration. The greater the decline due to the permutation of a single variable was, the greater the importance of this variable was relative to the other tested variables (Genuer et al., 2010). The most important independent environmental variable identified in the random forest analyses (average maximum night temperature) was then run in two separate models to determine the effects of (i) average maximum night temperature and treatment groups and (ii) the interaction with average maximum night temperature. Generalised linear models (negative binomial regressions) were run using $\mathrm{R}$ to assess the main effects and interactions. Recapture counts $(x)$ was used as the dependent variable, expressed as a fraction:

$x=(($ number of moths recaptured $) /($ number of moths released)).

From these two models, the best fit model with the lowest Akaike information criterion value (estimator of the relative quality of statistical models for a given data set) was chosen. Data were analysed as the total number of recaptures for both the cold-treated and control group at different average maximum night temperatures. The data distribution, degrees of freedom and residual deviance were examined and confirmed so that model assumptions were not violated.

\section{Results}

\section{Effects of cold treatment on laboratory flight activity}

The flight performance of $T$. leucotreta in the laboratory was affected by the interaction between treatment group and test 
Table 2 Summarised results of adult $T$. leucotreta laboratory flight performance assays

\begin{tabular}{|c|c|c|c|c|c|c|c|c|}
\hline \multirow[b]{2}{*}{ Effect } & \multicolumn{4}{|l|}{ Partial flyers } & \multicolumn{4}{|c|}{ Sustained flyers } \\
\hline & Coefficient & SE & Z-value & $P$-value & Coefficient & SE & Z-value & P-value \\
\hline Treatment & 0.236 & 0.579 & 0.406 & 0.684 & -1.882 & 0.914 & -2.057 & $<0.05$ \\
\hline Sex & -1.532 & 0.612 & -2.500 & $<0.05$ & -2.622 & 0.963 & -2.721 & $<0.01$ \\
\hline Test temperature & -1.173 & 0.637 & -1.840 & $<0.1$ & 0.637 & 0.818 & 0.777 & 0.436 \\
\hline Replicate & -0.228 & 0.238 & -0.955 & 0.774 & 0.149 & 0.287 & 0.517 & 0.604 \\
\hline Treatment: Sex & 0.274 & 0.433 & 0.632 & 0.527 & 0.192 & 0.605 & 0.316 & 0.751 \\
\hline Treatment: Test temperature & 2.002 & 0.431 & 4.642 & $<0.01$ & 3.268 & 0.662 & 4.931 & $<0.01$ \\
\hline Sex: Test temperature & 0.214 & 0.432 & 0.494 & 0.621 & 1.177 & 0.773 & 1.521 & 0.12 \\
\hline Treatment: Replicate & -0.672 & 0.266 & -2.526 & $<0.05$ & -0.088 & 0.334 & -0.264 & 0.791 \\
\hline Sex: Replicate & 0.666 & 0.259 & 2.561 & $<0.01$ & 0.186 & 0.339 & 0.548 & 0.583 \\
\hline Test temperature: Replicate & -0.072 & 0.267 & -0.268 & 0.788 & -0.613 & 0.369 & -1.660 & $<0.01$ \\
\hline
\end{tabular}

A multinomial model was used to test the effects of cold temperature treatment, test temperature, sex and replicate on the flight performance of adult moths. SE: Standard error of the mean. Text in bold indicates significant effects.

temperature as well as that between treatment, sex, test temperature and biological replicate. The interaction between treatment and test temperature was highly significant for both partial $(\mathrm{Z}=4.642 ; P<0.01 ; \mathrm{df}=1)$ and sustained flyers $(\mathrm{Z}=4.931$; $P<0.01 ; \mathrm{df}=1)$ (Table 2), whereas the biological replicate showed interactions with treatment $(\mathrm{Z}=-2.526 ; P<0.05$; $\mathrm{df}=1)$ and sex $(\mathrm{Z}=2.561 ; P<0.01 ; \mathrm{df}=1)$ for partial flyers compared with non-flyers. Treatment had a significant effect on sustained flyers $(Z=-2.057 ; P<0.05 ; \mathrm{df}=1)$ and the effect of sex was significant for both partial $(Z=-2.5 ; P<0.05 ; \mathrm{df}=1)$ and sustained flyers $(\mathrm{Z}=-2.721 ; P<0.01 ; \mathrm{df}=1)$. There was no significant interaction between treatment and sex, and test temperature and sex for the flight performance of adult $T$. leucotreta adults and biological replicate had no effect on their flying ability (Table 2).

Cold-treated males were more likely to fly under cooler laboratory conditions than were the control males (21 vs. $3 \%$ ) (Fig. 1). Under cold conditions, cold-treated males were also more prone to leave the Petri dish than the control males were. More than $80 \%$ of the control males did not fly from the Petri dish. When cold-treated males were released under warm conditions, they were less prone to fly than the control moths were, whereas nearly $40 \%$ of control moths successfully flew and another $20 \%$ were partial flyers (Fig. 1). Similar effects were seen in females (Fig. 1), although they were generally less prone to fly than males were, regardless of the temperature, whereas cold-treated females showed a greater attempt to fly under cold conditions, with up to $30 \%$ being partial flyers.

\section{Effects of cold treatment on field recapture counts}

The influence of different environmental parameters on recapture counts is shown Figure 2. The flight performance of $T$. leucotreta males in terms of recapture counts in the field was significantly influenced by the interaction between treatment and average maximum night temperature $(\mathrm{Z}=2.534, P<0.05$; $\mathrm{df}=28)$ and the effect of treatment $(\mathrm{Z}=-2.584, P<0.01 ; \mathrm{df}=28)$ as a single factor (Table 3 Fig. 2).

Clear differences were observed between the recapture counts of cold-treated and control moths over 3 days (Fig. 3A). The number of recaptured cold-treated males was higher than that

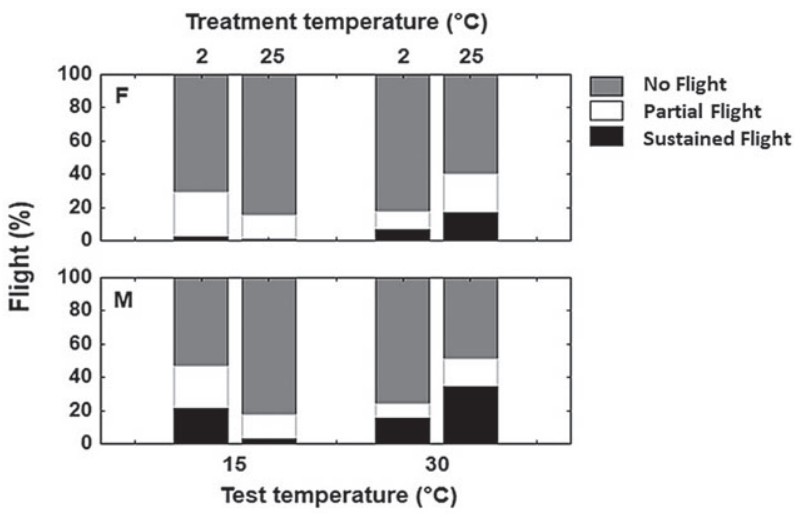

Figure 1 Results of laboratory flight performance assays of cold-treated $\left(2{ }^{\circ} \mathrm{C}\right)$ and control $\left(25^{\circ} \mathrm{C}\right) \mathrm{T}$. leucotreta female (F) and male (M) adults as measured at different test temperatures. Flight performance was scored according to three possible responses: Successful flight (sustained flight), landing in the surrounding water (partial flight) and non-dispersal (no flight).

of control moths (245 vs. 103) at average ambient night temperatures below $17^{\circ} \mathrm{C}$. As the average maximum night temperature decreased, the number of captured treated and control males decreased, but recapture counts of the latter were lower than those of the cold-treated group (Fig. 3A). For temperatures above $17^{\circ} \mathrm{C}$, cold-treated males had a poorer recapture count (432 vs. 688) than the control moths did. Therefore, most of the data points below $17^{\circ} \mathrm{C}$ that represent cold-treated moths were above the control line (Fig. 3B). Generally, the recapture counts of both groups were better when the average maximum night temperature was above $17^{\circ} \mathrm{C}$. Under these circumstances, control moths performed better than cold-treated moths did. However, during cold spells (temperatures below $17^{\circ} \mathrm{C}$ ), cold-treated males were recaptured in greater numbers than the control moths were. At $17^{\circ} \mathrm{C}$, there was no significant difference between the recapture counts of cold-treated and control moths, whereas at $15^{\circ} \mathrm{C}$, an increase of nearly $50 \%$ in the number of cold-treated moths relative to that of control moths was observed. At $11^{\circ} \mathrm{C}, 20$ control moths were caught, whereas 29 treated moths were recaptured, a difference of nearly $30 \%$. In contrast, the recapture counts of cold-treated moths at warm ambient night 


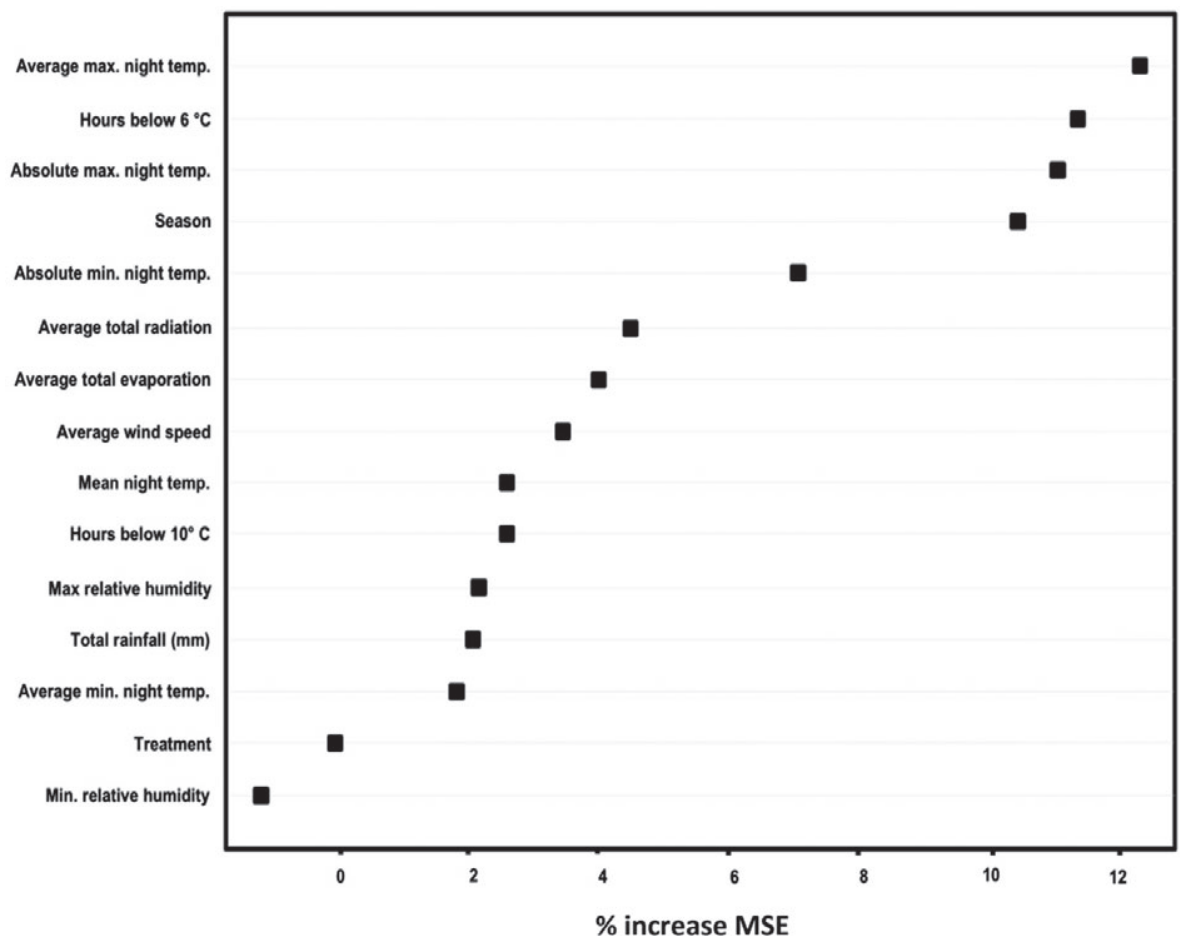

Figure 2 Results of random forest analyses, displaying the effect of different independent variables contributing to the flight performance of adult $T$. leucotreta scored as a percentage increase in the model's mean-squared error (MSE). The higher the MSE value is, the more important the variable is considered to be.

Table 3 Summarised results of a general linear model assessing flight performance (in terms of recapture counts) of adult T. leucotreta in field assays with average maximum night temperature, treatment and their interaction

\begin{tabular}{llll}
\hline & Estimate $\pm \mathrm{SE}$ & Z-value & $P$-value \\
\hline Intercept & $\mathbf{5 . 2 4 5} \pm \mathbf{1 . 4 4 6}$ & $\mathbf{3 . 6 2 6}$ & $<\mathbf{0 . 0 0 1}$ \\
$\begin{array}{l}\text { Average maximum night } \\
\quad \text { temperature }\end{array}$ & $-0.066 \pm 0.075$ & -0.883 & 0.377 \\
$\begin{array}{l}\text { Treatment } \\
\text { Average maximum } \\
\quad \text { night temperature: }\end{array}$ & $\mathbf{- 2 . 3 2 5 \pm 0 . 8 9 9}$ & $\mathbf{- 2 . 5 8 4}$ & $<\mathbf{0 . 0 1}$ \\
$\quad$ Treatment & & $\mathbf{2 . 5 3 4}$ & $<\mathbf{0 . 0 5}$ \\
\hline
\end{tabular}

SE: Standard error of the mean. Text in bold indicates significant relationships. Degrees of freedom $=28$.

temperatures were poorest, with $29 \%$ fewer cold-treated moths than control moths having been caught at $20^{\circ} \mathrm{C}$ and $53 \%$ fewer at $30^{\circ} \mathrm{C}$.

\section{Discussion}

Our results reveal that plastic physiological responses in adult T. leucotreta elicited during the cold treatment increased both their laboratory and field flight performance under cooler thermal conditions. The effects of cold treatment were more pronounced in the laboratory, whereas these effects were weaker, but nonetheless statistically significant, in the field. This is likely due to a greater number of factors influencing flight in the field.
The laboratory assays showed that almost $20 \%$ more cold-treated adult males took flight relative to control moths. Few moths took flight during the laboratory trials, but the overall tendency to fly was greater in the field. This is because only $40-50 \%$ of control moths attempted flight in the laboratory, even at warm temperatures. In addition, each flight assay in the laboratory allowed the moths only $2 \mathrm{~h}$ to fly from the release point. We suggest that, apart from the shorter exposure time, the reason for the lower flight performance in the laboratory may be that the fluorescent light was not as powerful an attractant as either real or artificial pheromones would be in the field. Moreover, in the field, there may also be important navigational or dispersal cues from horizon lines, polarised light, wind and pheromones, which may further promote dispersal (Drake, 1983; Cardé \& Willis, 2008).

As in the laboratory assays, the adult moth recapture count (i.e. abundance) from our field assays showed that cold-treated males were more likely to fly under cold conditions than control males were, with twice as many recaptures of the former at cooler temperatures (below $17^{\circ} \mathrm{C}$ ). This suggests that the efficacy of the SIT programme could be increased by treating adults below their CTmin for release during the winter or cold spells. Although recapture count in trap catches does not necessarily prove enhanced efficacy of an SIT programme, trap catches are considered an accepted, useful marker of field flight performance and are routinely assessed in SIT programmes (Chidawanyika \& Terblanche, 2011; Stringer et al., 2013). Furthermore, our results demonstrate the potential for thermal treatments to offset poor low-temperature performance of mass-reared moths and form a baseline for moving towards demonstrating SIT field 


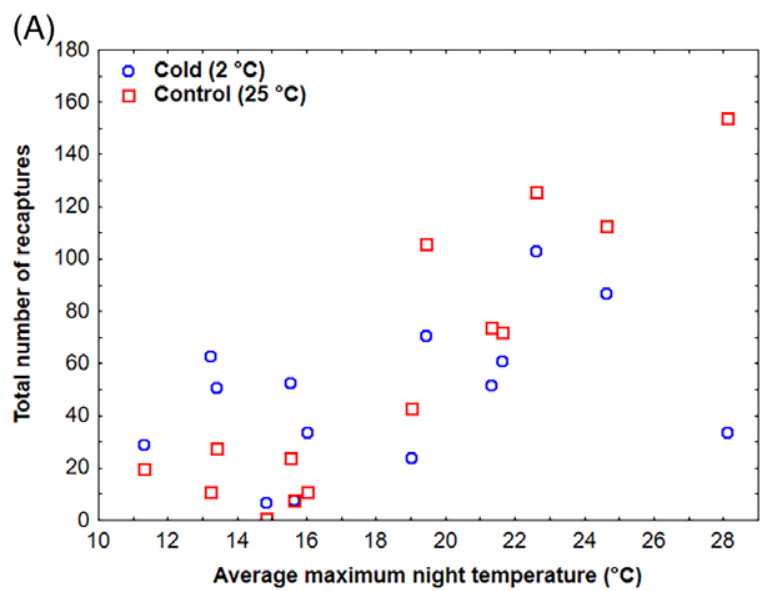

(B)

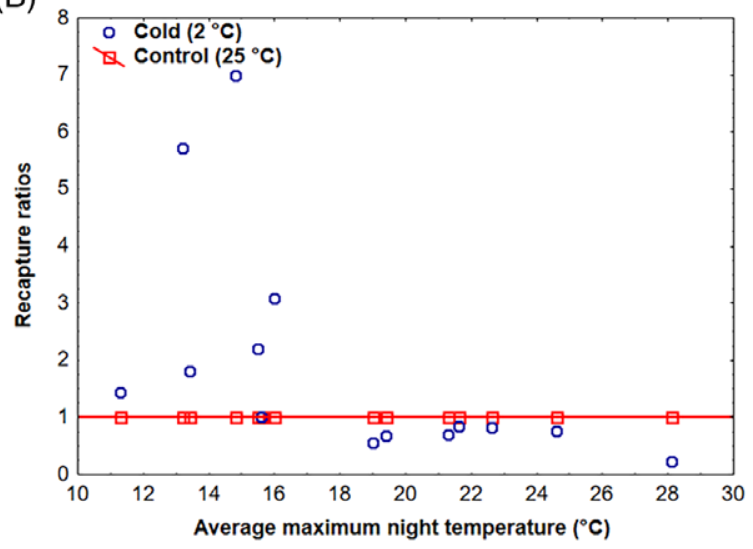

Figure 3 Field recapture numbers (male moths) of $T$. leucotreta used to estimate flight performance. Each data point represents the number moths recaptured on a specific release date. Recapture counts of moths are shown in relation to average maximum night temperatures either as absolute numbers (A) or as a ratio of treated moths relative to the control $\left(25^{\circ} \mathrm{C}\right)$ group, using the control moths as a baseline or reference level (B). [Colour figure can be viewed at wileyonlinelibrary.com].

efficacy. Further work is needed to validate other mating and fitness parameters in the field, such as competitive mating events, and reduction of the wild moth population under similar ambient conditions. Given our results, however, it can be argued that cold treatment provides mass-reared $T$. leucotreta with a significant advantage under cooler field temperatures and that this is a worthwhile advantage to pursue. Although this might complicate logistical planning for SIT programmes, such treatments could easily be incorporated during normal handling and transportation procedures.

Our results provide laboratory and field support for the beneficial acclimation hypothesis. In all cases, $T$. leucotreta performed best in environments simulating pre-release conditions (i.e. low temperatures) and poorer in environments to which they had not previously been exposed (i.e. warmer conditions) (Chidawanyika \& Terblanche, 2011; Kristensen et al., 2008; Loeschcke \& Hoffmann, 2007). This is particularly evident when flight performance of cold-treated moths released at low temperatures in the field is estimated as a ratio of the performance of control group moths (Fig. 3B). The benefits include better recapture counts and likely enhanced flight performance under cold conditions $\left(5-17^{\circ} \mathrm{C}\right)$. However, when cold-treated males were released at warmer ambient temperatures $\left(17-30^{\circ} \mathrm{C}\right)$, a decline in recapture counts was observed.

Similar effects were found in $C$. pomonella in which approximately twice as many cold-treated than control moths were recaptured under cooler ambient conditions if they had been acclimated at a low temperature in the laboratory prior to release (Chidawanyika \& Terblanche, 2011). However, these improvements in the flight performance of the cold-acclimated moths came at the cost of poorer performance in warmer environments (Chidawanyika \& Terblanche, 2011; Judd et al., 2004; Mateev et al., 2017). Another study on C. pomonella flight performance concluded that fluctuating rearing temperatures increased the flying ability of adult moths at cooler temperatures compared with that of moths reared at a constant temperature (Judd et al., 2011). Moreover, the recapture count after undergoing diapause of moths was higher but accompanied by a significant decrease in fecundity (Bloem et al., 2006; Chidawanyika \& Terblanche, 2011). Similar costs and benefits thermal effects were also seen across other species, such as Drosophila sp. (e.g. Loeschcke \& Hoffmann, 2007; reviewed in e.g. Terblanche, 2014).

Although improved recapture counts of mass-reared T. leucotreta during the warmer months were recorded when adults were not subjected to any cold temperature protocols, this is not a viable option for SIT programmes, as the moths will mate and injure themselves before being released into the field (Boersma $\&$ Carpenter, 2016). Moreover, such moths do not perform well under cold ambient conditions, which reduce the window for efficient recaptures in the winter (Stotter \& Terblanche, 2009). Comparable outcomes have been observed in C. pomonella. When compared with control adults, adults cold-treated prior to release dispersed better (e.g. over longer distances)(Judd et al., 2004; Mateev et al., 2017).

Boersma et al. (2018) found a difference between male and female performance (cold tolerance, fecundity and longevity) in T. leucotreta under different acclimation and treatment temperatures. Females demonstrated a greater response to environmental variation than males did and therefore exhibited a greater capacity for phenotypic plasticity in terms of CTmin, longevity and fecundity. However, this was not true for flight performance in this study, as males were more prone to fly than females were under varying environmental conditions. Nevertheless, this does not mean that females are less effective in SIT programmes under cooler climatic conditions, as they may still contribute to the programme's efficacy by attracting wild adult males.

The cellular and physiological mechanisms underlying the responses to different environmental conditions in $T$. leucotreta remain unclear. It can be argued that hormesis, whereby a beneficial effect results from some mild stress, through cold treatment as a mild stressor may mitigate any detrimental effects of radiation that potentially reduce moth fitness. Cold-treating moths after radiation may decrease indirect radiation damage or result in improved damage repair mechanisms upon rewarming, while the application of anaesthesia with the use of carbon dioxide or nitrogen could prevent injury (López-Martínez \& Hahn, 2012; López-Martínez et al., 2014). The use of anoxia (nitrogen) while sterilising lepidopterans has been shown to benefit flight performance in the cactus moth, Cactoblastis 
cactorum (Berg) (López-Martínez et al., 2014). Further work on this topic is required, as the cold treatment of T. leucotreta males after radiation has been found to lead to a poorer recapture count under warm environmental conditions, resulting in shorter flying distances than those of males not subjected to cold treatment (Boersma \& Carpenter, 2016). It is however unlikely that a loss of ion homeostasis explains poor performance post-chilling in T. leucotreta (Karsten et al., 2019).

In conclusion, our study demonstrated that plastic physiological responses to low temperature treatment leads to altered laboratory and field flight performance in T. leucotreta. The increased recapture count of sterile adults (of up to $50 \%$ more than control group moths) during the colder months will most probably benefit SIT programmes by increasing the sterile-to-wild moth ratio in the field, perhaps contributing to more successful mating of wild and mass-reared moths, that in turn may lead to a decline of the wild $T$. leucotreta population in the subsequent summer. Greater recapture counts in cooler months, resulting from improved flight performance of mass-reared, sterile and cold-treated $T$. leucotreta moths could lead to a progressive decline of the wild population, with each season starting with a smaller wild population, thereby increasing the overall efficacy of the SIT programme. This study provides evidence that low temperature treatment in the adult stage of mass-reared $T$. leucotreta could potentially improve the dispersal ability of sterile moths in SIT programmes, mitigating the reduced efficacy typically reported in cooler months.

\section{Acknowledgements}

Funding support was provided by Citrus Research International and XSIT (Pty) Ltd. LB and JST were supported by the South African National Research Foundation (NRF) and the NRF Centre of Excellence for Invasion Biology. JST was also supported by the International Atomic Energy Agency through a CRP on dormancy management of insects. We are grateful to the anonymous referees for constructive comments that helped improve the work.

\section{References}

Bloem, S., Bloem, K.A. \& Knight, A.L. (1998) Assessing the quality of mass-reared codling moths (Lepidoptera: Tortricidae) by using field release-recapture tests. Journal of Economic Entomology, 91, $1125-1130$.

Bloem, S., Carpenter, J.E., Bloem, K.A., Tomlin, L. \& Taggart, S. (2004) Effect of rearing strategy and gamma radiation on field competitiveness of mass-reared codling moths (Lepidoptera: Tortricidae). Journal of Economic Entomology, 97, 1891-1898.

Bloem, S., Carpenter, J. \& Dorn, S. (2006) Mobility of mass-reared diapaused and non-diapaused Cydia pomonella (Lepidoptera: Tortricidae): effect of different constant temperatures and lengths of cold storage. Journal of Economic Entomology, 99, 707-713.

Boersma, N. \& Carpenter, J.E. (2016) Influence of holding temperature and irradiation on field performance of mass-reared Thaumatotibia leucotreta (Lepidoptera: Tortricidae). Florida Entomologist, 99, 215-221.

Boersma, N., Boardman, L., Gilbert, M. \& Terblanche, J.S. (2018) Sex-dependent thermal history influences cold tolerance, longevity and fecundity in false codling moth, Thaumatotibia leucotreta (Lepidoptera: Tortricidae). Journal of Agricultural and Forest Entomology, 20, 41-50.
Cardé, R.T. \& Willis, M.A. (2008) Navigational strategies used by insects to find distant, wind-borne sources of odor. Journal of Chemical Ecology, 34, 854-866.

Chen, X. \& Ishwaran, H. (2012) Random forest for genomic data analysis. Genomics, 99, 232-329.

Chidawanyika, F. \& Terblanche, J.S. (2011) Costs and benefits of thermal acclimation for codling moth, Cydia pomonella (Lepidoptera: Tortricidae): implications for pest control and the sterile insect release programme. Evolutionary Applications, 4, 534-544.

Chown, S.L. \& Terblanche, J.S. (2007) Physiological diversity in insects: ecological and evolutionary contexts. Advances in Insect Physiology, 33, 150-152.

Colinet, H., Sinclair, B.J., Vernon, P. \& Renault, D. (2015) Insects in fluctuating thermal environments. Annual Review of Entomology, 60, $123-140$.

Denlinger, D. \& Lee, R. (2010) Low Temperature Biology of Insects. Cambridge University Press, New York.

Dowell, F.E., Parker, A.G., Benedict, M.Q., Robinson, A.A., Broce, A.B. \& Wirtz, R.A. (2005) Sex separation of tsetse fly pupae using near-infrared spectroscopy. Bulletin of Entomological Research, 95, 249-257.

Drake, V.A. (1983) Collective orientation by nocturnally migrating Australian plague locusts, Chortoicetes terminifera (Walker) (Orthoptera: Acrididae): a radar study. Bulletin of Entomological Research, 73, 679-692.

Enserink, M. (2007) Proven technology may get a makeover. Science, 317, 312-313.

Esterhuizen, N., Clusella-Trullas, S., Van Daalen, C.E., Schoombie, R.E., Boardman, L. \& Terblanche, J.S. (2014) Effects of within-generation thermal history on the flight performance of Ceratitis capitata: colder is better. Journal of Experimental Biology, 217, 3545-3556.

Fasolo, A.G. \& Krebs, R.A. (2004) A comparison of behavioural change in Drosophila during exposure to thermal stress. Biological Journal of the Linnean Society, 83, 197-205.

Fay, H.A.C. \& Meats, A. (1987) The sterile insect release method and the importance of thermal conditioning before release: field-cage experiments with Dacus tryoni in spring weather. Australian Journal of Zoology, 35, 197-204.

Field, A., Miles, J. \& Field, Z. (2012) Discovering Statistics Using R. Sage, London.

Fletcher, B.S. \& Zervas, G. (1977) Acclimation of different strains of the olive fruit fly, Dacus oleae, to low temperatures. Journal of Insect Physiology, 23, 649-653.

Genuer, R., Poggi, J. \& Tuleau-Malot, C. (2010) Variable selection using random forests. Pattern Recognition Letters, 31, 2225-2236.

Hofmeyr, J.H., Carpenter, J.E. \& Bloem, S. (2005) Developing the sterile insect technique for Cryptophlebia leucotreta (Lepidoptera: Tortricidae): influence of radiation dose and release ratio on fruit damage and population growth infield cages. Journal of Economic Entomology, 98, 1924-1929.

Hofmeyr, J.H., Carpenter, J.E., Bloem, S., Slabbert, J.P., Hofmeyr, M. \& Groenewald, S.S. (2015) Development of the sterile insect technique to suppress false codling moth Thaumatotibia leucotreta (Lepidoptera: Tortricidae) in citrus fruit: research to implementation (part 1). African Entomology, 23, 180-186.

Ju, X., Grego, C. \& Zhang, X. (2013) Specific effects of fiber size and fiber swelling on biomass substrate surface area and enzymatic digestibility. Bioresource Technology, 144, 232-239.

Judd, G.J.R., Gardiner, M.G.T. \& Thistlewood, H.M.A. (2004) Seasonal variation in recapture of mass-reared sterile codling moth, Cydia pomonella (Lepidoptera: Tortricidae): implications for control by sterile insect technique in British Columbia. Journal of the Entomological Society of British Columbia, 101, 29-43.

Judd, G.J.R., Arthur, S., Deglow, K. \& Gardiner, M.G.T. (2011) Operational mark-release-recapture field tests comparing competitiveness 
of wild and differentially mass-reared codling moths from the Okanagan-Kootenay sterile insect program. The Canadian Entomologist, 143, 300-316.

Judd, G.J.R. \& Gardiner, M.G.T. (2005) Towards eradication of codling moth in British Colombia by complimentary actions of mating disruption, tree banding and sterile insect technique: five-year study in organic orchards. Crop Protection, 24, 718-733.

Judd, G.R.J. \& Gardiner, M.G.T. (2006) Temperature, irradiation and delivery as factors affecting spring-time flight activity and recapture of mass-reared male codling moths released by the Okanagan-Kootenay sterile insect programme. Journal of the Entomological Society of British Columbia, 103, 19-32.

Karsten, M., Lebenzon, J.E., Sinclair, B.J., Terblanche, B.J. (2019) Loss of ion homeostasis is not the cause of chill coma or impaired dispersal in false codling moth Thaumatotibia leucotreta (Lepidoptera: Tortricidae). Comparative Biochemistry and Physiology Part A: Molecular \& Integrative Physiology, 229, 40-44.

Klassen, W. \& Curtis, C.S. (2005) History of the sterile insect technique. Sterile Insect Technique: Principles and Practice in Area-Wide Integrated Pest Management (ed. by V. A. Dyck, J. Hendrichs, and A. S. Robinson), pp. 3-36. Springer, Dordrecht.

Krafsur, E.S. (1998) Sterile insect technique for suppressing and eradicating insect population: 55 years and counting. Journal of Agricultural Entomology, 15, 303-313.

Kristensen, T.N., Hoffmann, A.A., Overgaard, J., Sørensen, J.G., Hallas, R. \& Loeschcke, V. (2008) Costs and benefits of cold acclimation in field-released Drosophila. Proceedings of the National Academy of Sciences of the United States of America, 105, 216-221.

Leroi, A.M., Bennett, A.F. \& Lenski, R.E. (1994) Temperature acclimation and competitive fitness: an experimental test of the beneficial acclimation assumption. Proceedings of the National Academy of Sciences of the United States of America, 91, 1917-1921.

Liaw, A. \& Wiener, M. (2002) Classification and regression by randomForest. R News, 2, 18-22.

Lindquist, D.A., Abusowa, M. \& Hall, M.J.R. (1992) The New World screwworm fly in Libya: a review of its introduction and eradication. Medical and Veterinary Entomology, 6, 2-8.

López-Martínez, G., Carpenter, J.E., Hight, S.D. \& Hahn, D.A. (2014) Low-oxygen atmospheric treatment improves the performance of irradiation-sterilized male cactus moths used in SIT. Journal of Economic Entomology, 107, 155-197.

López-Martínez, G. \& Hahn, D.A. (2012) Short-term anoxic conditioning hormesis boosts antioxidant defenses, lowers oxidative damage following irradiation and enhances male sexual performance in the Caribbean fruit fly, Anastrepha suspense. Journal of Experimental Biology, 215, 2150-2161.

Loeschcke, A. \& Hoffmann, A.A. (2007) Consequences of heat hardening on a field fitness component in drosophila depend on environmental temperature. The American Naturalist, 169, 175-183.

Mateev, E., Kwon, J.J., Judd, G.J.R. \& Evenden, M.L. (2017) The effect of cold storage of mass-reared codling moths (Lepidoptera: Tortricidae) on subsequent flight capacity. The Canadian Entomologist, 149, 391-398.

Meats, A. (1976) Developmental and long-term acclimation to cold by the Queensland fruit-fly (Dacus tryoni) at constant and fluctuating temperatures. Journal of Insect Physiology, 22, 1013-1019.
Parker, A.G. (2005) Mass-rearing for sterile insect release. Sterile Insect Technique: Principles and Practice in Area-Wide Integrated Pest Management (ed. by V. A. Dyck, J. Hendrichs, and S. A. Robinson), pp. 209-232. Springer, Dordrecht.

Powell, S.J. \& Bale, J.S. (2005) Low temperature acclimated populations of the grain aphid Sitobion avenae retain ability to rapidly cold harden with enhanced fitness. Journal of Experimental Biology, 208, 2615-2620.

Qian, S.S., Cuffney, T.F. \& McMahon, G. (2012) Multinomial regression for analyzing macroinvertebrate assemblage composition data. Freshwater Science, 31, 681-694.

Sgrò, C.M., Terblanche, J.S. \& Hoffmann, A.A. (2016) What can plasticity contribute to insect responses to climate change? Annual Review of Entomology, 61, 433-451.

Shreve, S.M., Kelty, J.D. \& Lee, R.E. Jr. (2004) Preservation of reproductive behaviours during modest cooling: rapid cold-hardening fine-tunes organismal response. Journal of Experimental Biology, 207, $1797-1802$.

Simmons, G.S., Suckling, D.M., Carpenter, J.E., Addison, M.F., Dyck, V.A. \& Vreysen, M.J.B. (2010) Improved quality management to enhance the efficacy of the sterile insect technique for lepidopteran pests. Journal of Applied Entomology, 134, 261-273.

Sørensen, J.G., Addison, M.F. \& Terblanche, J.S. (2012) Mass-rearing of insects for pest management: challenges, synergies and advances from evolutionary physiology. Crop Protection, 38, 87-94.

Stotter, R.L. \& Terblanche, J.S. (2009) Low-temperature tolerance of false codling moth Thaumatotibia leucotreta (Meyrick) (Lepidoptera: Tortricidae) in South Africa. Journal of Thermal Biology, 34, $320-325$.

Stringer, L.D., Sullivan, N.J., Sullivan, T.E.S. et al. (2013) Attractiveness and competitiveness of irradiated light brown apple moths. Entomologia Experimentalis et Applicata, 148, 203-212.

Teets, N.M. \& Denlinger, D.L. (2013) Physiological mechanisms of seasonal and rapid cold-hardening in insects. Physiological Entomology, 38, 105-116.

Terblanche, J.S. (2014) Physiological performance of field-released insects. Current Opinion in Insect Science, 4, 60-64.

Terblanche, J.S. \& Chown, S.L. (2007) Factory flies are not equal to wild flies. Science, 317, 1678-1682.

Terblanche, J.S., Mitchell, K.A., Uys, W., Short, C. \& Boardman, L. (2017) Thermal limits to survival and activity in two life stages of false codling moth Thaumatotibia leucotreta (Lepidoptera, Tortricidae). Physiological Entomology, 42, 379-388.

Vreysen, M.J.B. \& Robinson, A.S. (2010) Ionising radiation and area-wide management of insect pests to promote sustainable agriculture: a review. Agronomy for Sustainable Development, 31, 233-250.

Weldon, C.W., Terblanche, J.S. \& Chown, S.L. (2011) Time-course for attainment and reversal of acclimation to constant temperature in two Ceratitis species. Journal of Thermal Biology, 36, 479-485.

Wilson, R.S. \& Franklin, C.E. (2002) Testing the beneficial acclimation hypothesis. Trends in Ecology \& Evolution, 17, 66-70.

Woods, H.A. \& Harrison, J.F. (2002) Interpreting rejections of the beneficial acclimation hypothesis: when is physiological plasticity adaptive? Evolution, 56, 1863-1866.

Accepted 22 January 2019

First published online 21 February 2019 